\title{
Graphene Doping Reaches New Levels
}

\author{
New experiments with doped graphene take the two-dimensional \\ material beyond its "Van Hove singularity" to regions that may host exotic \\ states of matter.
}

By Michael M. Scherer

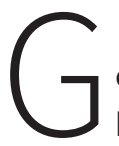
raphene is famous for its peculiar electronic properties exemplified by the Dirac point, a region in the material's band structure where electron behavior resembles that of high-energy particles. An electron carrying charge through graphene in a Dirac-like fashion acts like a single particle that barely interacts with its many peers. Now, Philipp Rosenzweig and his colleagues from the Max Planck Institute for Solid State Research in Germany, have added an excessive amount of charge carriers to graphene [1], moving the material away from said Dirac point to a Van Hove point and, reportedly for the first time, even beyond. The Van Hove point features a large number of states, which gives charge carriers ample opportunity to interact, conspire, and form collective states of matter, such as magnetism and superconductivity. Such collective states cannot be described in a single-particle picture. Excitingly, theoretical predictions for doped graphene include exotic states, like high-temperature chiral topological superconductivity [2], which can now be explored with the controllable doping techniques demonstrated by Rosenzweig and colleagues.

Electrons in crystalline solids populate multiple energy bands. In two-dimensional materials like graphene, these energy bands feature points where the electronic density of states diverges-a Van Hove singularity [3]. Such a large concentration of states enhances the effects of many-body interactions and supports the formation of a collective state of matter. But which collective state is uncertain, as there is a subtle competition at the Van Hove singularity between magnetism and superconductivity (as well as other ordered phases), with the "winner" depending on the precise shape of the energy bands.
This type of competition may help explain the observed superconductivity in high-temperature superconductors [4] and magic-angle bilayer graphene [5] (see Trend: Bilayer Graphene's Wicked, Twisted Road). In the Van Hove scenario for unconventional superconductors, it is argued that repulsive interactions between electrons cause fluctuations-in spin, for example-that, in turn, induce attractive interactions [6]. These attractive components can act as a glue between electrons, facilitating the formation of Cooper pairs and, hence, superconductivity. Testing this scenario is one of the main motivations for doping graphene to high levels.

Graphene is a promising candidate for studying Van Hove superconductivity, as its hexagonal symmetry has been argued to tip the balance between magnetism and superconductivity decisively in favor of the latter. In pristine monolayer graphene, the Fermi level, which characterizes the highest filled electron energy levels at zero temperature, is relatively far (a few electron volts) away from the Van Hove point. However, the Van Hove point can be reached by chemically doping the material by, for example, intercalation. Here, atoms of a certain type are inserted between the graphene monolayer and a substrate, resulting in a transfer of charge carriers from the intercalated atoms to graphene. Depending on the choice of atoms for the intercalation, the amount of transferred charge is different. Previous studies have reported that such chemical doping techniques-using calcium [7] and gadolinium [8] for the intercalation atoms-can bring the Fermi level in graphene near the Van Hove point.

In their new experiment Rosenzweig and co-workers studied 


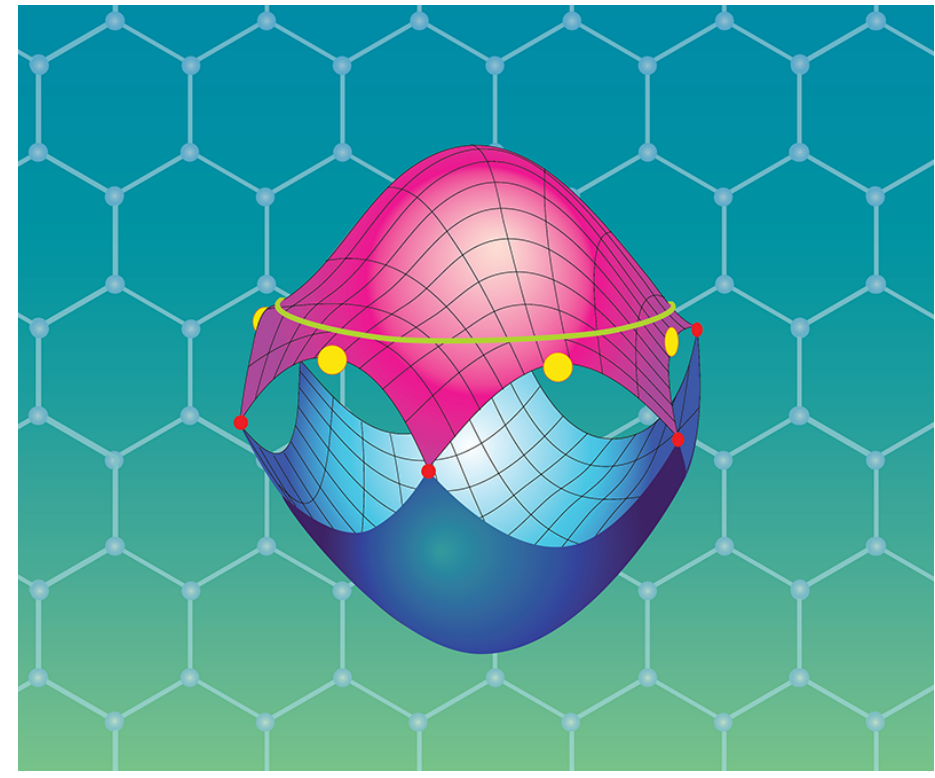

Figure 1: The electronic band structure of graphene exhibits a so-called Van Hove singularity, where the density of states diverges. This divergence occurs at multiple Van Hove points (yellow dots) that are at higher energy than the well-known Dirac points (red dots). As graphene is doped, the Fermi level (green line) moves up to the Van Hove singularity and beyond, resulting in enhanced many-body effects that can produce collective states such as unconventional forms of superconductivity and magnetism. Credit: APS/Carin Cain

the doping of a graphene monolayer by intercalation with ytterbium. To analyze the results of the doping procedure in terms of the electron band structure, they used angle-resolved photoemission spectroscopy (ARPES) with high angular resolution and at low temperatures of about $20 \mathrm{~K}$. They observed that the charge transfer between ytterbium and graphene caused the energy bands of graphene to fill up, moving the Fermi level from the Dirac point to the Van Hove point. The photoemission data also revealed that the conducting energy band of graphene is strongly warped into a flat band extending over a wide range of crystal momenta.

Further, the Fermi level is pinned to the Van Hove point, yielding an extended Van Hove singularity. These band features-seen previously in studies of doped graphene $[7,8]$ and of high-temperature superconductors-cannot be explained with a single-particle picture but instead require that many-body interactions are at work.
However, the researchers did not stop at the Van Hove singularity. They provided additional charge transfer onto the graphene sheet by deposition of potassium. This hybrid-doping procedure led to a further increase of the charge-carrier density, eventually depinning the Fermi level from the Van Hove singularity. The result is a topological change in the shape of the Fermi surface, which is the contour in momentum space where electron energy is at the Fermi level. This shape change, or Lifshitz transition, is characterized by the merging of two filled regions (electron pockets) into one big empty region (hole pocket). These phenomena are evidence that Rosenzweig and colleagues have, for the first time, coaxed a graphene monolayer into the regime of overdoping, providing a controllable procedure to experimentally tune the doping in a sizeable range near Van Hove filling.

These doping techniques now set the stage for a systematic exploration of graphene near the Van Hove point. The effects of many-body interactions that are expected here will not just modify the electronic band structure but will also lead to even more severe changes of the system as correlated phases emerge. Previous theoretical considerations-indicating a tilt towards superconductivity over magnetism and other orders in the graphene case [4] - did not take into account the observed reconstructed band structure, which still requires a full theoretical understanding on its own. A first model study suggests that the modified Fermi surface may mix up the competition by adding other ordered phases to the list of contenders, possibly including novel types of spin or charge order [9]. Moreover, the extended Van Hove singularity might lead to an even higher density of states than expected, which could result in a higher transition temperature for the superconducting state. What sort of superconductivity might Van-Hove-doped graphene host? The answer might be chiral topological superconductivity [10], which holds considerable promise for future technological applications in, for example, quantum computing. Therefore, an exciting time lies ahead, where future experiments can be expected to complement or challenge our understanding of collective states of matter.

Michael M. Scherer: Institute for Theoretical Physics, University of Cologne, Cologne, Germany

\section{REFERENCES}

1. P. Rosenzweig et al., "Overdoping graphene beyond the Van 
Hove singularity,” Phys. Rev. Lett. 125, 176403 (2020).

2. R. Nandkishore et al., "Chiral superconductivity from repulsive interactions in doped graphene," Nat. Phys. 8, 158 (2012).

3. L. Van Hove, "The occurrence of singularities in the elastic frequency distribution of a crystal," Phys. Rev. 89, 1189 (1953).

4. H. Isobe et al., "Unconventional superconductivity and density waves in twisted bilayer graphene," Phys. Rev. X 8, 041041 (2018).

5. S. Maiti and A. V. Chubukov, "Superconductivity from repulsive interaction," AIP Conf. Proc. 1550, 3 (2013).

6. K. Le Hur and T. M. Rice, "Superconductivity close to the Mott state: From condensed-matter systems to superfluidity in optical lattices," Ann. Phys. (N.Y.) 324, 1452 (2009).

7. J. L. McChesney et al., "Extended Van Hove singularity and superconducting instability in doped graphene," Phys. Rev. Lett. 104, 136803 (2010).

8. S. Link et al., "Introducing strong correlation effects into graphene by gadolinium intercalation," Phys. Rev. B 100, 121407 (2019).

9. L. Classen et al., "Competing orders at higher-order Van Hove points," Phys. Rev. B 102, 125141 (2020).

10. A. M. Black-Schaffer, "Edge properties and Majorana fermions in the proposed chiral $d$-wave superconducting state of doped graphene," Phys. Rev. Lett. 109, 197001 (2012). 